\title{
LOW TEMPERATURE GEOTHERMAL RESOURCE EVALUATION OF THE MOSES LAKE-RITZVILLE-CONNELL AREA, WASHINGTON
}

By

Scott Widness

November 1983

Work Performed Under Contract No. AC07-79ET27014

Washington State University

Pullman, Washington

Technical Information Center Office of Scientific and Technical Information United States Department of Energy 


\section{DISCLAIMER}

This report was prepared as an account of work sponsored by an agency of the United States Government. Neither the United States Government nor any agency Thereof, nor any of their employees, makes any warranty, express or implied, or assumes any legal liability or responsibility for the accuracy, completeness, or usefulness of any information, apparatus, product, or process disclosed, or represents that its use would not infringe privately owned rights. Reference herein to any specific commercial product, process, or service by trade name, trademark, manufacturer, or otherwise does not necessarily constitute or imply its endorsement, recommendation, or favoring by the United States Government or any agency thereof. The views and opinions of authors expressed herein do not necessarily state or reflect those of the United States Government or any agency thereof. 


\section{DISCLAIMER}

Portions of this document may be illegible in electronic image products. Images are produced from the best available original document. 


\title{
DISCLAIMER
}

\begin{abstract}
This report was prepared as an account of work sponsored by an agency of the United States Government. Neither the United States Government nor any agency thereof, nor any of their employees, makes any warranty, express or implied, or assumes any legal liability or responsibility for the accuracy, completeness, or usefulness of any information, apparatus, product, or process disclosed, or represents that its use would not infringe privately owned rights. Reference herein to any specific commercial product, process, or service by trade name, trademark, manufacturer, or otherwise does not necessarily constitute or imply its endorsement, recommendation, or favoring by the United States Government or any agency thereof. The views and opinions of authors expressed herein do not necessarily state or reflect those of the United States Government or any agency thereof.
\end{abstract}

This report has been reproduced directly from the best available copy.

Available from the National Technical Information Service, U. S. Department of Commerce, Springfield, Virginia 22161.

Price: Printed Copy A03

Microfiche A01

Codes are used for pricing all publications. The code is determined by the number of pages in the publication. Information pertaining to the pricing codes can be found in the current issues of the following publications, which are generally available in most libraries: Energy Research Abstracts (ERA); Government Reports Announcements and Index (GRA and 1); Scientific and Technical Abstract Reports (STAR); and publication NTIS-PR-360 available from NTIS at the above address. 
STATE OF WASHINGTON

DEPARTMENT OF NATURAL RESOURCES

BRIAN J. BOYLE, Commissioner of Public Lands

JAMES A. STEARNS, Department Supervisor

DIVISION OF GEOLOGY AND EARTH RESOURCES

Raymond Lasmanis. State Geologist

LOW TEMPERATURE GEOTHERMAL RESOURCE EVALUATION

OF THE MOSES LAKE-RITZVILLE-CONNELL AREA, WASHINGTON

November 1983

by

Scott Widness

Department of Civil and Environmental Engineering

Geological Engineering Section

Washington State University

Pullman, Washington 99164

Open-File Report 83-11

Prepared under U.S. Department of Energy

Contract No. DE-AC07-79ET27014

for Assessment of Geothermal Resources in Washington 
The study area is located in portions of Adams. Grant. Lincoln. and Frankl in counties of eastern Washington (figures 1 and 2 ). The area is representative of a complex stratigraphic and geohydrologic system within the basalt flows of the Columbia River Basalt Group. The regional piezometric surface and stratigraphic units dip towards the southwest (Luzier and Burt, 1974: Tanaka. 1979).

Fluid temperature data were collected by three different agencies. The Geological Engineering Section (WSU) at Washington State University. runs a continuous fluid temperature (FT) $10 \mathrm{~g}$ as part of a complete suite of geophysical logs. The U.S. Geological Survey (USGS) runs a continous fluid FT $\log$ in conjunction with caliper and natural-gama logs. Southern Methodist University (SMU) and the Washington State Department of Natural Resources, Division of Geology and Earth Resources (DNR). have cooperated in gathering FT data. The DNR-SMU data were collected by taking temperature measurements at $5 \mathrm{~m}$ intervals. Bottom-hole temperatures (BHT) and bottom-hole depths (BHD) of selected wells in the study area are given in table 2. Some of the BHT data in table 2 may vary from those previously reported by WSU. These discrepancies are the result of changes in the calibration method of the FT tool.

A technique developed by Biggane (1982) was used to determine the geothermal gradients within the area. A least squares linear regression analysis of the relationship between the BHT and BHD was used to determine. the geothermal gradient of a given well data group (WDG). Well data groups were selected on the premises of geographic proximity, position within the regional groundwater flow system, land slope azimuth. and land slope dip (figure 2). Some data points have been excluded from the linear regression analysis on the basis of factors such as duplicate logging of the same hole, down-hole flow, holes not logged to total depth, and questionable FT tool responses. 


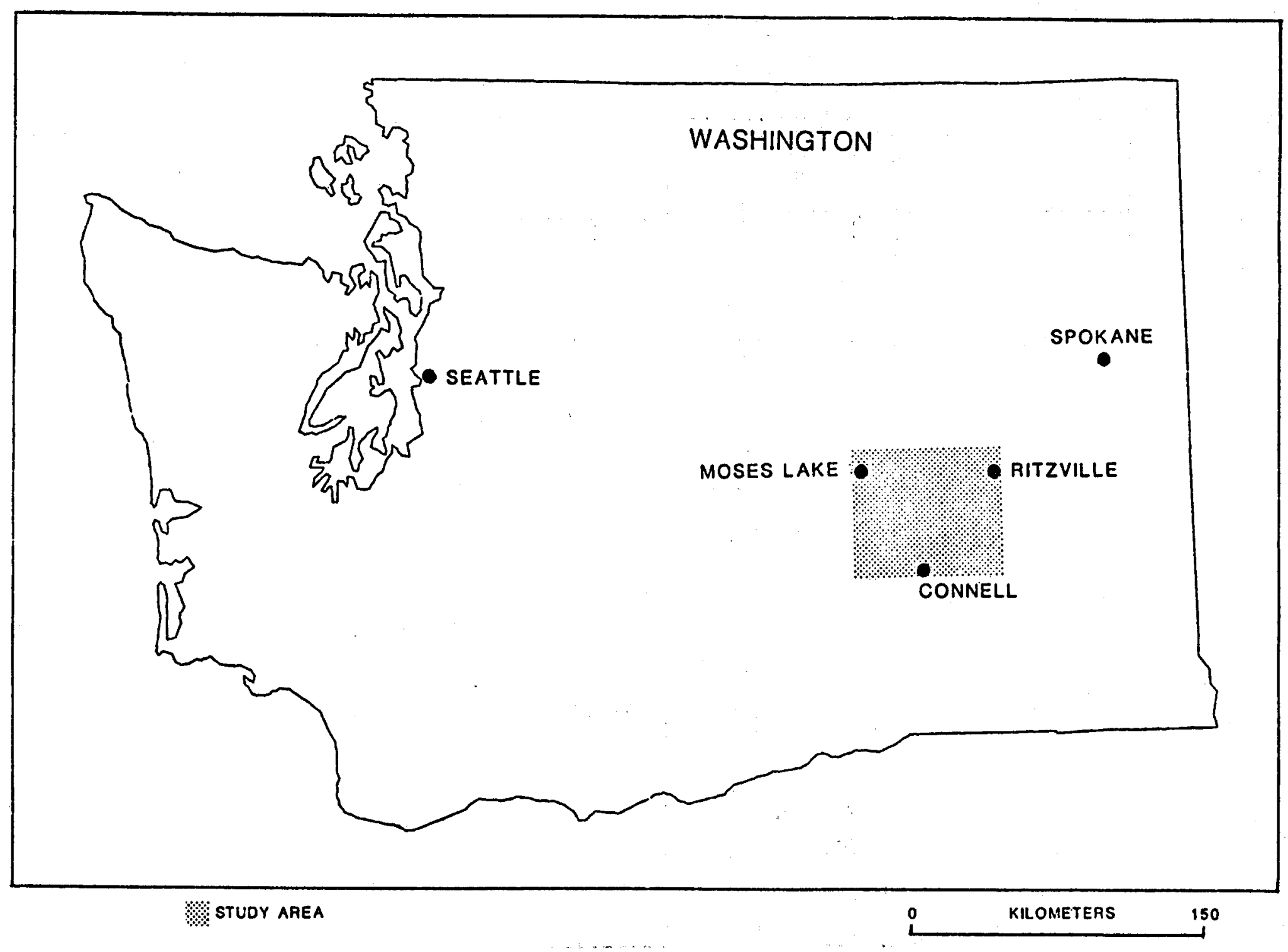

FIGURE 1 LOCATION OF THE STUDY AREA 


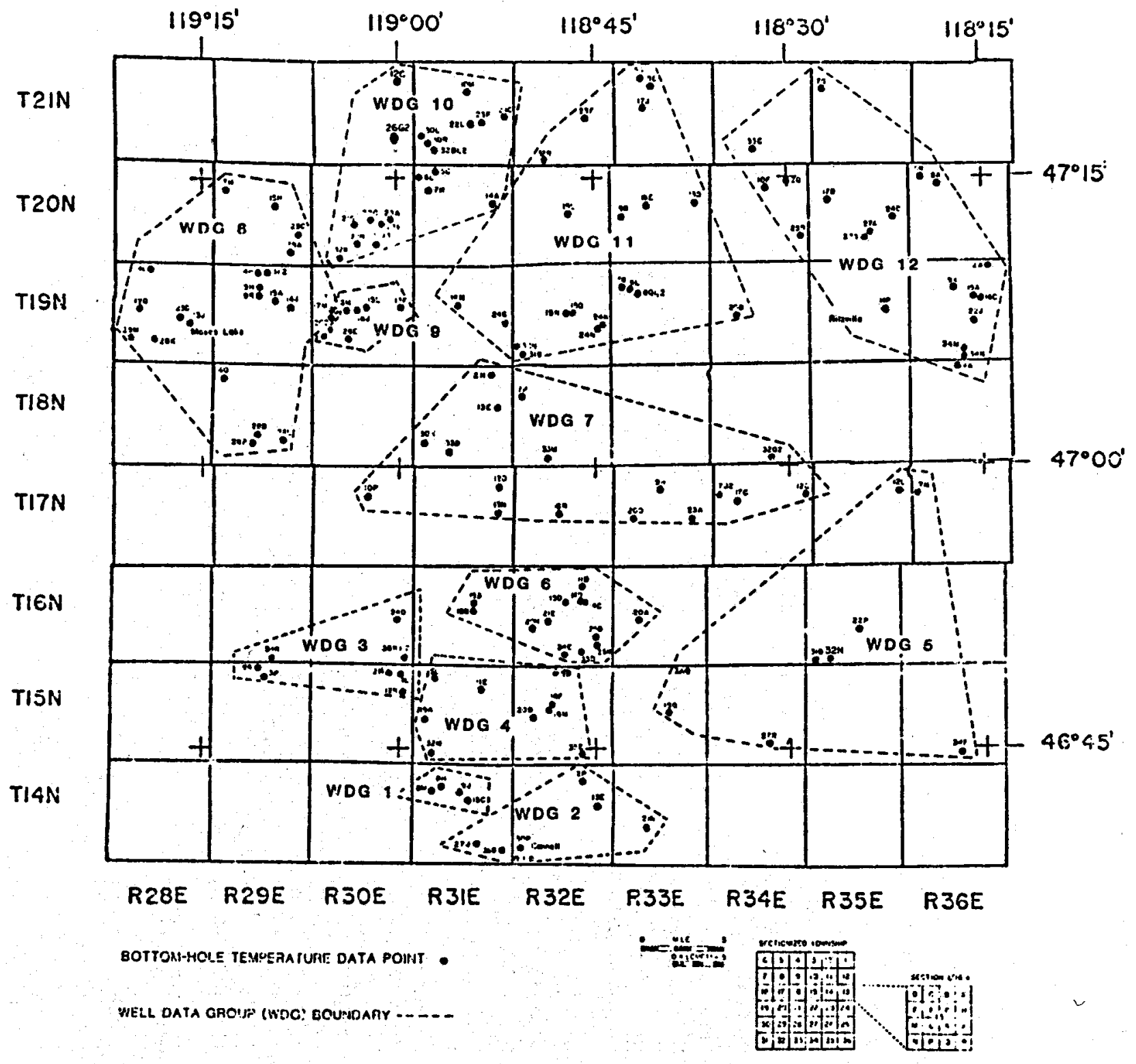

FIGURE 2 LOCATION OF BOTTOM-HOLE TEMPERATURE DATA 
Table 2. - LOCATIONS, DESIGNATIONS, BOTTOM-HOLE TEMPERATURES, AND BOTTOM-HOLE DEPTHS OF SELECTED WELLS

Source Location Owner Date $\underset{(\mathrm{m})}{\text { Elev. }} \underset{(\mathrm{m})}{\text { Depth }} \underset{\left({ }^{\circ} \mathrm{C}\right)}{\mathrm{BHT}} \quad$ WDG Comuents

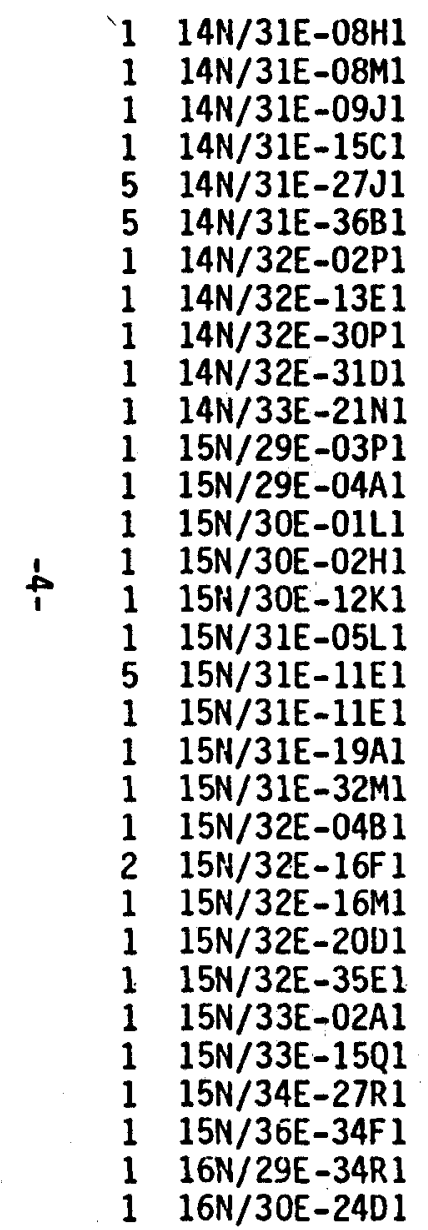

Rathbun, Corrin

Rathbun, Corrin

Rathbun, Corrin, \#3

$01 / 20 / 77$

333.8

196.3

$16.0 \quad 1$

Kummer Farms

Andrews, Clyde

$07 / 18 / 80$

$11 / 13 / 79$

$04 / 29 / 71$

Connell City

Hart, Frank

$04 / 20 / 71$

$06 / 20 / 79$

Hart, Dick

$11 / 10 / 71$

Connell City \#7

Connell City \#6

Heider, Walter

$06 / 18 / 76$

$01 / 20 / 72$

$02 / 15 / 75$

Othello City \#5

Othello City \#6

Matthews, Clyde \#1

Matthews, Ciyde, \#2

Meldrin, Ted

Mckay, Ed

Lyle Bros.

Lyle Enterprises \#3

Johnson, Arthur

Kummer, Clarence. \#4

$05 / 06 / 74$

$02 / 15 / 79$

$12 / 11 / 74$

$12 / 09 / 74$

$02 / 12 / 76$

$05 / 19 / 76$

$10 / 26 / 71$

$09 / 05 / 73$

$05 / 11 / 75$

Damon, Don

Stelger

Hatton Test \#1 (DNR)

$03 / 20 / 80$

$11 / 09 / 71$

$04 / 03 / 79$

Hart, Cyril

Tompkins, Robert

$09 / 03 / 71$

Strohmaier, Albert

$05 / 24 / 72$

$03 / 15 / 74$

Wats on, Leroy

Blauert, Fred

$11 / 30 / 72$

Othello City \#3

$03 / 23 / 72$

K1 iphardt, G. W.

$01 / 06 / 77$

347.5

342.9

339.9

387.1

275.6

376.4

386.2

265.2

259.4

423.7

320.7

321.6

387.7

379.5

382.5

379.5

381.0

381.0

378.6

344.4

381.0

758.0

43.1

22.2

413.3

207.3

20.3

$84.1 \quad 15.5$

$235.6 \quad 27.1$

$232.2 \quad 23.7$

$252.4 \quad 17.8$

$302.3 \quad 29.0$

351.128 .2

$298.4 \quad 29.4$

$367.3 \quad 24.7$

216.6

278.0

408.7

6.4

14.8

$404.4 \quad 26.8$

$213.3 \quad 20.1$

$211.5 \quad 19.0$

$341.7 \quad 27.6$

$364.5 \quad 26.0$

$266.2 \quad 21.9$

381.0

179.8

335.6

365.8

376.4

452.6

438.6

455.7

323.1

340.5

214.9

307.8

252.1

202.4

242.6

213.0

260.0

20.0

15.2

18.2

22.9

24.9

18.1

19.9

25.3

219.826 .1

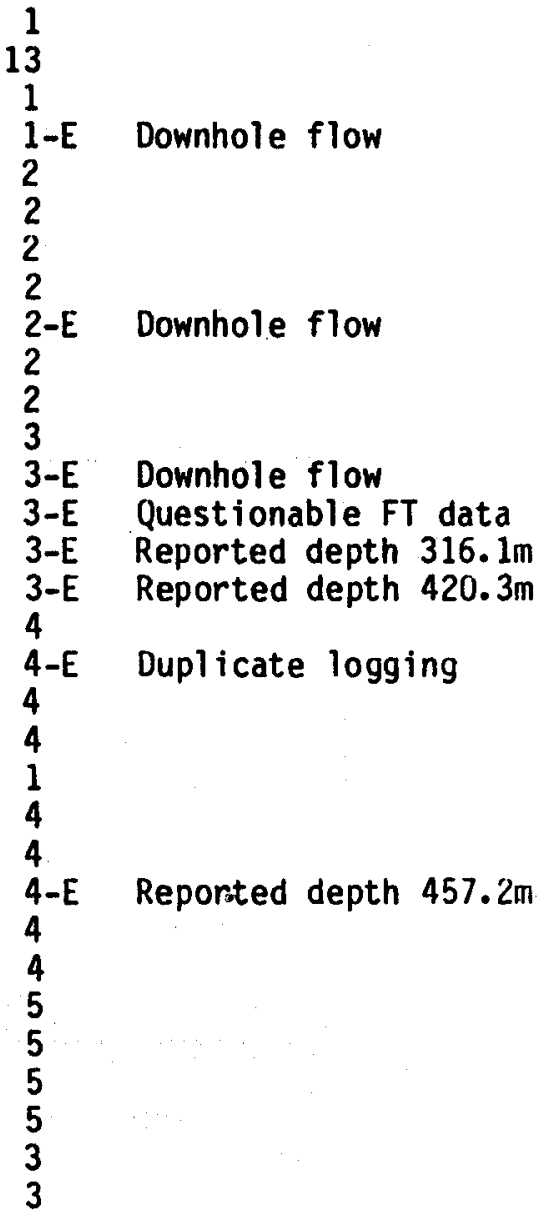


Table 2. - LOCATIONS, DESIGNATIONS, BOTTOM-HOLE TEMPERATURES, AND BOTTOM-HOLE DEPTHS OF SELECTED WELLS (Cont'd)

Source Location Owner Date Elev. Depth BHT WDG Comments

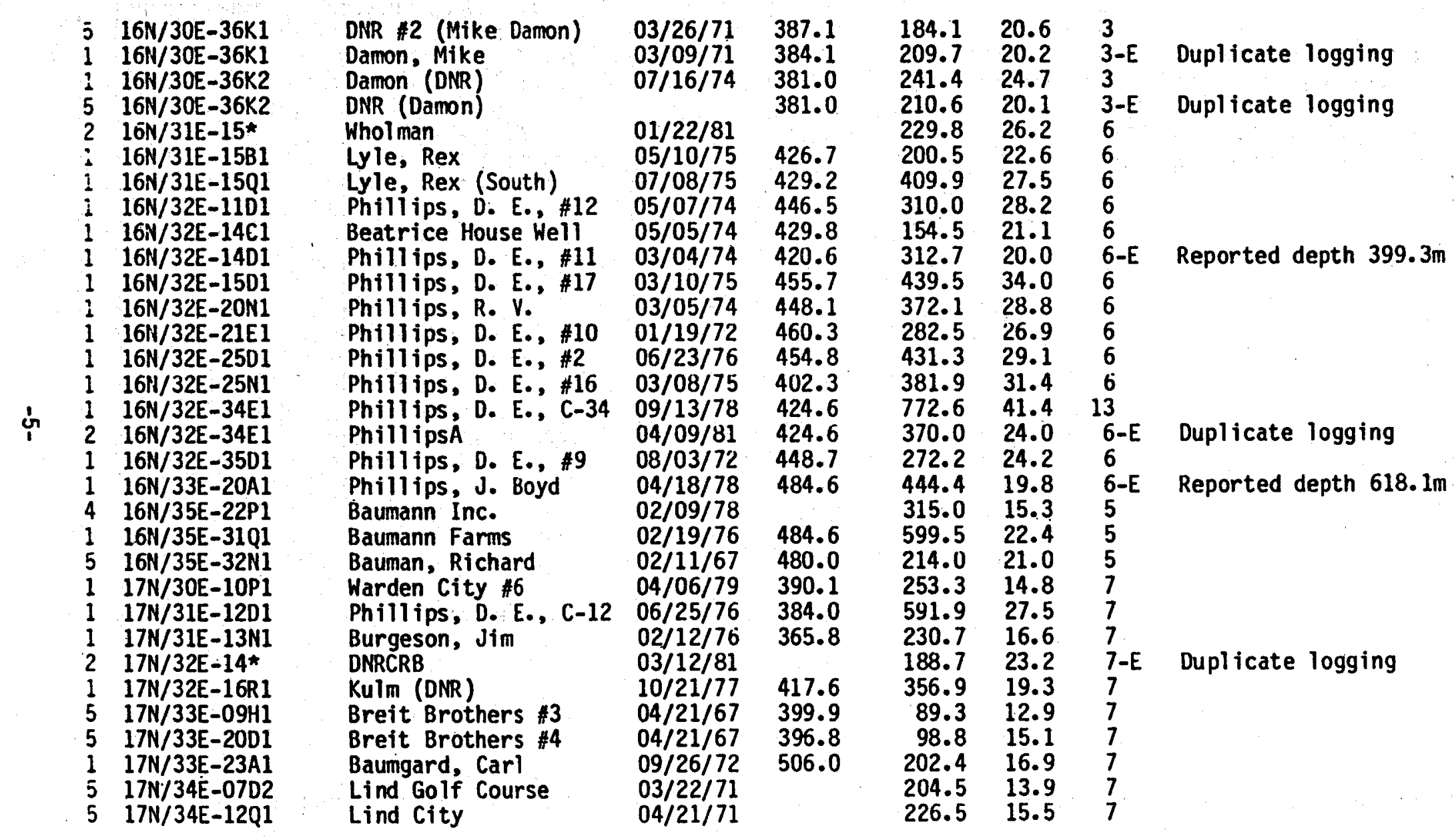


Table 2. - LOCATIONS, DESIGNATIONS, BOTTOM-HOLE TEMPERATURES, AND BOTTOM-HOLE DEPTHS OF SELECTED WELLS (CONT'd)

\begin{tabular}{|c|c|c|c|c|c|c|c|}
\hline ource Location & Owner & Date & $\begin{array}{c}\text { Elev. } \\
\text { (m) }\end{array}$ & $\begin{array}{l}\text { Depth } \\
\text { (m) }\end{array}$ & $\begin{array}{l}\mathrm{BHT} \\
\left({ }^{\circ} \mathrm{C}\right)\end{array}$ & WDG & Comients \\
\hline $\begin{array}{ll}5 & 17 N / 34 E-17 C 1 \\
1 & 17 N / 35 E-12 L 1 \\
1 & 17 N / 36 E-07 N 1 \\
1 & 18 N / 29 E-06 Q 1 \\
5 & 18 N / 29 E-26 L 1 \\
5 & 18 N / 29 E-28 B 1 \\
5 & 18 N / 29 E-28 P 1 \\
5 & 18 N / 31 E-02 H 1 \\
5 & 18 N / 31 E-13 E 1 \\
1 & 18 N / 31 E-30 K 1 \\
1 & 18 N / 31 E-33 D 1 \\
5 & 18 N / 32 E-07 P 1 \\
3 & 18 N / 32 E-33 M 1 \\
5 & 18 N / 34 E-32 Q 2 \\
1 & 18 N / 36 E-04 A 1 \\
3 & 19 N / 28 E-04 L 1 \\
2 & 19 N / 28 E-1701 \\
1 & 19 N / 28 E-23 D 1 \\
1 & 19 N / 28 E-23 J 1 \\
1 & 19 N / 28 E-28 K 1 \\
5 & 19 N / 28 E-28 K 4 \\
5 & 19 N / 28 E-29 M 1 \\
1 & 19 N / 28 E-29 M 1 \\
1 & 19 N / 29 E-03 F 2 \\
1 & 19 N / 29 E-04 H 1 \\
1 & 19 N / 29 E-09 H 1 \\
5 & 19 N / 29 E-09 H 1 \\
1 & 19 N / 29 E-14 J 1 \\
1 & 19 N / 29 E-15 A 1 \\
5 & 19 N / 30 E-13 F 1 \\
1 & 19 N / 30 E-15 L 1 \\
1 & 19 N / 30 E-16 J 1\end{array}$ & $\begin{array}{l}\text { Phillips, Chester } \\
\text { Kison, Rachel } \\
\text { Kautz, William B. } \\
\text { American Potato \#2 } \\
\text { A\&W Feedlot } \\
\text { Landis Farms \#6 } \\
\text { Land is Farms } \\
\text { Hutterian Bretheren } \\
\text { Franz, Victor } \\
\text { Dyck, Leo } \\
\text { Phili ips, D. E., C-33 } \\
\text { Hutterian Bretheren } \\
\text { Franz } \\
\text { WSU Exp. Sta. } \\
\text { Heinemann, Don } \\
\text { Moses Lake } \\
\text { Dunkin } \\
\text { Moses Lake City \#7 } \\
\text { Moses Lake City \#5 } \\
\text { Moses Lake City \#4 } \\
\text { Moses Lake \#4 } \\
\text { City of Westlake } \\
\text { Moses Lake City \#31 } \\
\text { Fode, Roy, \#2 } \\
\text { Shinn, Frink, \#2 } \\
\text { Shinn, Frank, \#1 } \\
\text { Shinn Irrigation } \\
\text { Jett-Aero \#2 } \\
\text { Masto Farms } \\
\text { American Potato } \\
\text { Radach, Jerry } \\
\text { Sparks, Dave, (East) }\end{array}$ & $\begin{array}{l}08 / 18 / 67 \\
12 / 16 / 71 \\
09 / 19 / 78 \\
01 / 23 / 75 \\
03 / 04 / 71 \\
04 / 02 / 71 \\
04 / 02 / 71 \\
03 / 05 / 71 \\
08 / 13 / 67 \\
12 / 07 / 76 \\
08 / 12 / 76 \\
11 / 11 / 67 \\
11 / 15 / 81 \\
02 / 13 / 67 \\
02 / 19 / 76 \\
08 / 25 / 81 \\
01 / 12 / 81 \\
09 / 27 / 74 \\
09 / 28 / 74 \\
09 / 26 / 74 \\
03 / 08 / 72 \\
05 / 19 / 71 \\
03 / 04 / 75 \\
01 / 16 / 76 \\
03 / 03 / 75 \\
01 / 30 / 73 \\
02 / 27 / 73 \\
03 / 03 / 74 \\
11 / 26 / 74 \\
02 / 15 / 67 \\
12 / 08 / 74 \\
12 / 07 / 74\end{array}$ & $\begin{array}{l}428.2 \\
499.9 \\
527.3 \\
355.1 \\
341.4 \\
355.1 \\
341.4 \\
371.8 \\
429.8 \\
396.9 \\
432.8 \\
396.2 \\
498.3 \\
539.2\end{array}$ & $\begin{array}{r}91.4 \\
135.6 \\
274.3 \\
204.8 \\
91.1 \\
97.5 \\
61.0 \\
122.5 \\
299.3 \\
188.1 \\
727.5 \\
239.9 \\
319.0 \\
184.7 \\
281.0 \\
221.5 \\
163.7 \\
282.2 \\
210.3 \\
293.5 \\
294.7 \\
141.7 \\
140.2 \\
322.2 \\
280.7 \\
175.3 \\
175.3 \\
218.2 \\
288.6 \\
202.1 \\
359.6 \\
279.2\end{array}$ & $\begin{array}{l}12.7 \\
19.3 \\
13.3 \\
21.7 \\
14.7 \\
16.7 \\
15.0 \\
13.0 \\
15.6 \\
15.0 \\
30.2 \\
21.7 \\
19.2 \\
16.2 \\
16.3 \\
19.3 \\
15.9 \\
23.8 \\
17.7 \\
16.2 \\
17.5 \\
19.8 \\
20.6 \\
29.0 \\
25.7 \\
14.1 \\
11.8 \\
21.5 \\
21.1 \\
20.6 \\
16.6 \\
15.9\end{array}$ & $\begin{array}{c}7 \\
5 \\
5 \\
8 \\
8 \\
8 \\
8 \\
7 \\
7 \\
7 \\
7 \\
7 \\
7 \\
7 \\
12-\mathrm{E} \\
8 \\
8 \\
8 \\
8-\mathrm{E} \\
8-\mathrm{E} \\
8-\mathrm{E} \\
8-\mathrm{E} \\
8-\mathrm{E} \\
8 \\
8 \\
8 \\
8-\mathrm{E} \\
8 \\
8 \\
9 \\
9 \\
9\end{array}$ & $\begin{array}{l}\text { Reported depth } 289.5 \mathrm{~m} \\
\text { Reported depth } 304.8 \mathrm{~m} \\
\text { Duplicate logging } \\
\text { Duplicate logging } \\
\text { Reported depth } 210.3 \mathrm{~m}\end{array}$ \\
\hline
\end{tabular}


Table 2. - LOCATIONS, DESIGNATIONS, BOTTOM-HOLE TEMPERATURES, AND BBOTTOM-HOLE DEPTHS OF SELECTED WELLS (CONT'd)

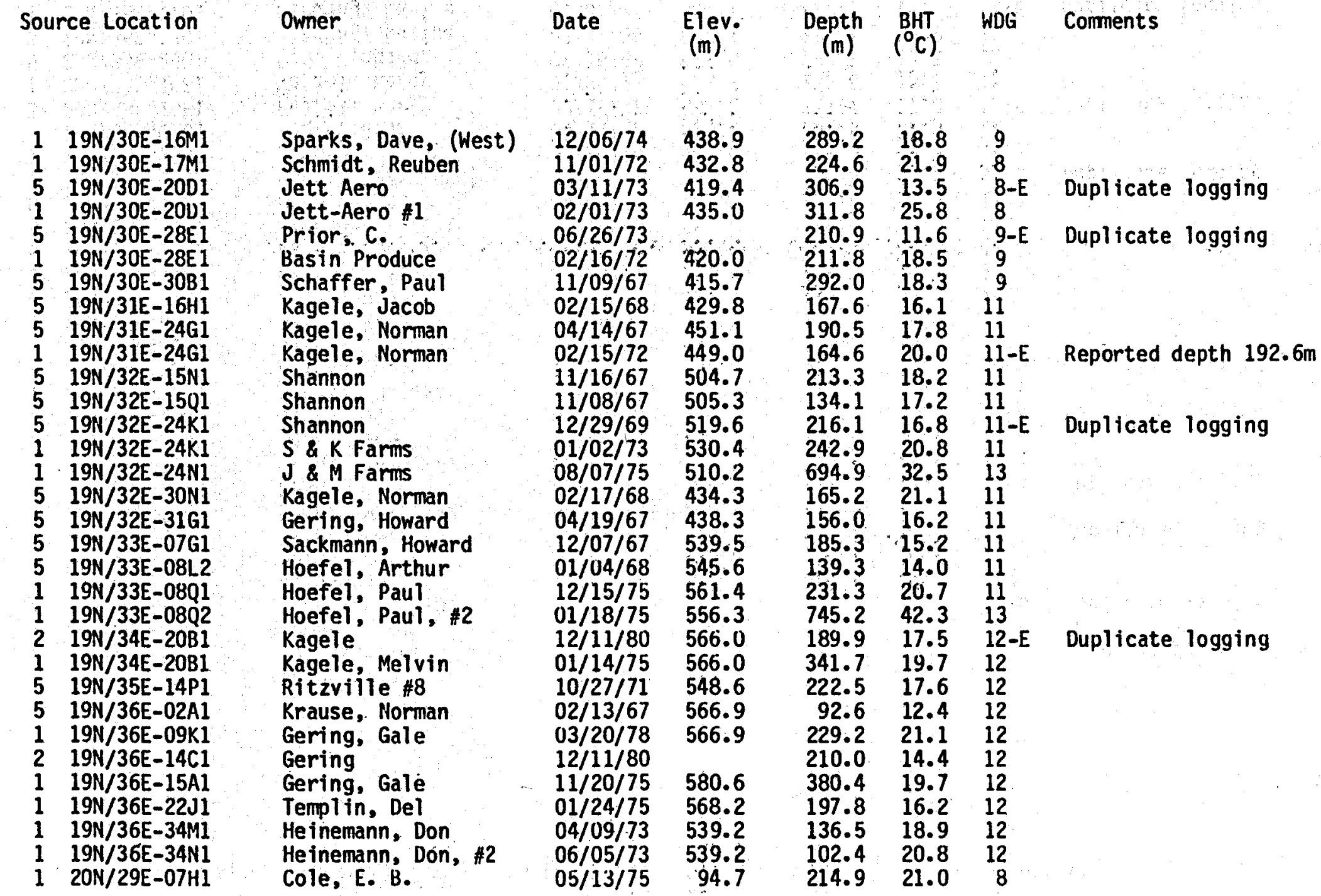


Table 2. - LOCATIONS, DESIGNATIONS, BOTTOM-HOLE TEMPERATURES, AND BOTTOM-HOLE DEPTHS OF SELECTED WELLS (CONT'd)

\begin{tabular}{|c|c|c|c|c|c|c|c|}
\hline Source Location & Owner & Date & $\underset{(m)}{\text { Elev. }}$ & $\begin{array}{l}\text { Depth } \\
(\mathrm{m})\end{array}$ & $\left({ }^{\mathrm{BHT}} \mathrm{C}\right)$ & WDG & Comments \\
\hline $\begin{array}{ll}1 & 20 N / 29 E-15 H 1 \\
1 & 20 N / 29 E-25 C 1 \\
1 & 20 N / 29 E-35 A 1 \\
5 & 20 N / 30 E-21 G 1 \\
1 & 20 N / 30 E-21 G 1 \\
5 & 20 N / 30 E-22 C 1 \\
5 & 20 N / 30 E-23 A 1 \\
1 & 20 N / 30 E-23 A 1 \\
5 & 20 N / 30 E-23 E 1 \\
1 & 20 N / 30 E-23 E 1 \\
5 & 20 N / 30 E-27 J 3 \\
5 & 20 N / 30 E-28 R 1 \\
1 & 20 N / 30 E-28 R 1 \\
1 & 20 N / 30 E-32 K 1 \\
1 & 20 N / 31 E-05 C 1 \\
5 & 20 N / 31 E-06 L 1 \\
5 & 20 N / 31 E-07 H 1 \\
5 & 20 N / 31 E-14 A 1 \\
5 & 20 N / 32 E-15 L 1 \\
5 & 20 N / 33 E-1301 \\
5 & 20 N / 33 E-16 E 1 \\
1 & 20 N / 33 E-16 E 1 \\
1 & 20 N / 33 E-19 B 1 \\
1 & 20 N / 34 E-0241 \\
5 & 20 N / 34 E-10 F 1 \\
1 & 20 N / 34 E-10 F 1 \\
5 & 20 N / 34 E-25 G 1 \\
5 & 20 N / 35 E-17 D 1 \\
1 & 20 N / 35 E-17 D 1 \\
1 & 20 N / 35 E-2401 \\
1 & 20 N / 35 E-27 A 1 \\
5 & 20 N / 35 E-27 G 1 \\
& 201 \\
1 & \end{array}$ & $\begin{array}{l}\text { Cole, Ivan } \\
\text { Reinke Farms } \\
\text { Powers, Tom } \\
\text { Claassen, Clint } \\
\text { Claassen, Clint } \\
\text { Spics, Herb } \\
\text { Franz, Herb } \\
\text { Franz, Herb, \#2 } \\
\text { Franz, Herb } \\
\text { Franz, Herb, \#l } \\
\text { Jantz, Joe } \\
\text { Jantz, Joe } \\
\text { Stucky, J. Jantz } \\
\text { Neibaur/West } \\
\text { Kissler, Merl in } \\
\text { Frick, Fred } \\
\text { Frick, David } \\
\text { Krell, Miller } \\
\text { Ragust, W. C. } \\
\text { Schoonover, Otto } \\
\text { USGS Odessa Test } \\
\text { Odessa Test (DOE) } \\
\text { Schorzman, Ray } \\
\text { Heber, John } \\
\text { Weizel, Leroy } \\
\text { Weizel, Leroy } \\
\text { Ott, Richard } \\
\text { Hardung, Lavine } \\
\text { Hardung, Lavine } \\
\text { Ahern, Cliff } \\
\text { Kagele, Richard } \\
\text { Kagele, Richard }\end{array}$ & $\begin{array}{l}01 / 31 / 73 \\
01 / 21 / 76 \\
05 / 12 / 75 \\
05 / 30 / 67 \\
12 / 28 / 77 \\
01 / 28 / 71 \\
02 / 14 / 73 \\
02 / 13 / 73 \\
03 / 30 / 72 \\
03 / 22 / 72 \\
02 / 06 / 67 \\
04 / 20 / 72 \\
04 / 12 / 72 \\
02 / 13 / 76 \\
01 / 06 / 72 \\
11 / 05 / 67 \\
05 / 14 / 71 \\
03 / 04 / 71 \\
04 / 27 / 67 \\
02 / 29 / 68 \\
04 / 05 / 71 \\
03 / 04 / 71 \\
08 / 29 / 74 \\
04 / 11 / 72 \\
02 / 15 / 72 \\
03 / 06 / 72 \\
02 / 16 / 68 \\
03 / 02 / 68 \\
09 / 04 / 71 \\
05 / 04 / 74 \\
01 / 07 / 77 \\
08 / 12 / 67\end{array}$ & $\begin{array}{l}416.1 \\
433.4 \\
408.4 \\
474.9 \\
475.5 \\
485.8 \\
500.2 \\
500.2 \\
484.6 \\
484.0 \\
484.6 \\
466.3 \\
464.8 \\
445.0 \\
501.4 \\
484.9 \\
481.6 \\
456.6 \\
521.8 \\
527.3 \\
509.0 \\
507.5 \\
541.0 \\
594.4 \\
585.2 \\
583.7 \\
573.0 \\
604.1 \\
604.1 \\
592.8 \\
611.1 \\
591.3\end{array}$ & $\begin{array}{l}158.2 \\
405.7 \\
292.6 \\
322.8 \\
469.7 \\
123.7 \\
219.4 \\
218.5 \\
337.1 \\
335.9 \\
176.8 \\
181.3 \\
178.3 \\
382.5 \\
172.5 \\
187.1 \\
158.8 \\
132.0 \\
237.7 \\
155.1 \\
228.0 \\
225.5 \\
238.0 \\
201.5 \\
237.7 \\
236.8 \\
189.3 \\
232.5 \\
227.1 \\
157.0 \\
364.8 \\
242.0\end{array}$ & $\begin{array}{l}14.3 \\
26.3 \\
26.6 \\
25.3 \\
28.7 \\
15.8 \\
19.3 \\
21.9 \\
34.9 \\
26.6 \\
15.3 \\
28.6 \\
20.3 \\
21.1 \\
19.0 \\
16.0 \\
13.8 \\
13.6 \\
14.8 \\
18.2 \\
15.9 \\
14.5 \\
19.5 \\
21.0 \\
17.8 \\
18.3 \\
13.3 \\
20.9 \\
15.3 \\
20.6 \\
15.6 \\
13.7\end{array}$ & $\begin{array}{l}8 \\
8 \\
8 \\
10-E \\
10 \\
10 \\
10-E \\
10 \\
10-E \\
10 \\
10 \\
10-E \\
10 \\
10-E \\
10 \\
10 \\
10 \\
10 \\
11 \\
11 \\
11 \\
11-E \\
11 \\
12 \\
12-E \\
12 \\
12 \\
12 \\
12-E \\
12 \\
12-E \\
12\end{array}$ & $\begin{array}{l}\text { Reported depth } 413.0 \mathrm{~m} \\
\text { Duplicate logging } \\
\text { Duplicate logging } \\
\text { Duplicate loyging } \\
\text { Duplicate logging } \\
\text { Reported depth } 399.3 \mathrm{~m}\end{array}$ \\
\hline
\end{tabular}


Table 2. - LOCATIONS., DESIGNATIONS, BOTTOM-HOLE, TEMPERATURES, AND BOTTOM-HOLE DEPTHS OF SELECTED WELLS (CONT'd)

Source Location

20N/36E-U6R1

20N/36E-08A1

21N/3OE-12C1

21N/30E-26G2

21N/31E-10M1

21N/31E-10M1

$21 N / 31 E-22 L 1$

$21 N / 31 E-22 L 1$

$21 \mathrm{~N} / 31 \mathrm{E}-23 \mathrm{~F} 1$

21N/31E-23F1

$21 N / 31 E-24 C 1$

$21 N / 31 E-30 L 1$

21N/31E-3OR1

5 21N/31E-30R 1

$6 \quad 121 N / 31 E-32 D 1$

$121 N / 31 E-3202$

$121 N / 32 E-23 F 1$

5 2IN/32E-32R1

$521 N / 33 E-05 Q 1$

1 21N/33E-09E1

5 21N/33E-17J1

$521 N / 34 E-33 C$

$121 N / 34 E-33 C 1$

5 21N/35E-07G1
Owner

Curtis, Ed

Curtis, Bill

Beck

Sche11, Harvey

Basalt Explorer \#1

DABE-1

Bàtes, Don, \#2

Bates, Don, \#2

Walter, Hayne

Schafer, Jerry

Schibel, Don

Kissler, Bob

Kissler, Bob

Kissler, Fred

Kissler, Fred

Kissler, Bob

Schafer, Jerry

Fink, Al

Odessa \#2

Odessa City

Rami, Emi I

Hardung, Joe

Hardung, Joe

Iverson, Bill
Date

$02 / 27 / 68$

$04 / 21 / 69$

$02 / 19 / 68$

$11 / 12 / 67$

$06 / 14 / 72$

$08 / 31 / 61$

$04 / 17 / 67$

$09 / 23 / 76$

$12 / 22 / 67$

$12 / 13 / 73$

$02 / 07 / 71$

$03 / 20 / 73$

$03 / 19 / 73$

$12 / 20 / 67$

$02 / 14 / 72$

$04 / 03 / 77$

$02 / 01 / 72$

$02 / 08 / 67$

$05 / 12 / 71$

$11 / 22 / 77$

$12 / 21 / 67$

$04 / 24 / 67$

03/09/69

$11 / 20 / 66$
Elev

584.3
581.9
499.9
490.7
490.7
514.5
533.4
540.1
552.3
536.4
512.1
502.0
502.0
508.7
509.0
552.3
527.9
472.1
490.7
543.7
585.8
585.2
612.0

Depth $\stackrel{\text { BHT }}{\text { B }}$ (m) WDG

135.9
144.8

231.0

171.0

1340.1

1249.7

233.8

477.9

226.8

292.9

193.8

143.2

263.3

236.5

209.4

365.7

297.2

192.6

75.6

201.2

207.3

270.6

241.7

134.7

14.212

13.712

13.310

$21.4 \quad 10$

$64.5 \quad 13$

$19.8 \quad 10$

$16.7 \quad 10$

21.010

$20.6 \quad 10$

$15.9 \quad 10$

23.8 10

$19.6 \quad 10$

$18: 9 \quad 10$

$23.8 \quad 11$

$17.2 \quad 11$

$10.9 \quad 11$

14.511

18.211

$24.7 \quad 12$

$15.6 \quad 12$
57.5 13-E Duplicate logging

$17.8: 10-E$ Duplicate logging

17.1 10-E Duplicate logging

17.2 11-E Duplicate logging

*Location unknown

Source:

1) Washington State University, Geological Engineering.

2) Kane, John R., Washington State Departinent of Natural Resources Division of Geoloyy and Earth Resources, 0lympia, WA.

3) Barker, Washington State Departinent of Natural Resources Divisoin of Geology and Earth Resources, Olympia, WA.

4) Blackwell, David D., 1979, Heat Flow and Geothermal gradient Measurements in in Washingt on to 1979 and Temperature-Depth Data Collected During 1979. Washington Departinent of Natural Resources Division of Geology and Earth Resources Olympia Washington.

5) U.S. Geological Survey (USGS) Tacoma, Washington, Unpublished logs. 
Data points which have excluded are followed by an "E" in figures 3 through 15 and in the table 2. The results of the analysis are given in table 1 and figures 3 through 15.

Table 1

Projected

Surface

WDG Temperature Gradient

$\begin{array}{rrr}1 & 7.1^{\circ} \mathrm{C}+47.9^{\circ} \mathrm{C} / \mathrm{Km} \\ 2 & 13.0 \mathrm{C}+49.9 \mathrm{C} / \mathrm{Km} \\ 3 & 9.4 \mathrm{C}+65.3 \mathrm{C} / \mathrm{Km} \\ 4 & 9.1 \mathrm{C}+49.1 \mathrm{C} / \mathrm{Km} \\ 5 & 917.1 \mathrm{C}+31.9 \mathrm{C} / \mathrm{Km} \\ 6 & 10.4 \mathrm{C}+26.8 \mathrm{C} / \mathrm{Km} \\ 7 & 10.8 \mathrm{C}+45.0 \mathrm{C} / \mathrm{Km} \\ 8 & 11.3 \mathrm{C}+35.0 \mathrm{C} / \mathrm{Km} \\ 9 & 9.7 \mathrm{C}+40.1 \mathrm{C} / \mathrm{Km} \\ 10 & 13.4 \mathrm{C}+21.9 \mathrm{C} / \mathrm{km} \\ 11 & 13.4 \mathrm{C}+38.1 \mathrm{C} / \mathrm{Km}\end{array}$

WDG's 1 through 4 indicate the highest gradients and are most promising for further investigation into the use of low-temperature goethermal resources. A qualitative assessment can be made of each WDG by comparing the number of scattered data points to the number of data points which lie on the calculated gradient.

It would be misleading to calculate a geothermal gradient for WDG 5 . The sparse distribution of data over a large area is primarily responsible. The inconsistency of data in Figure 7 (WDG 5) implies that several potential WDG's exist over the area, but there are not enough data to support their delineation. 


\section{BOTTOM-HOLE TEMPERATURE $\left({ }^{\circ} \mathrm{C}\right)$}

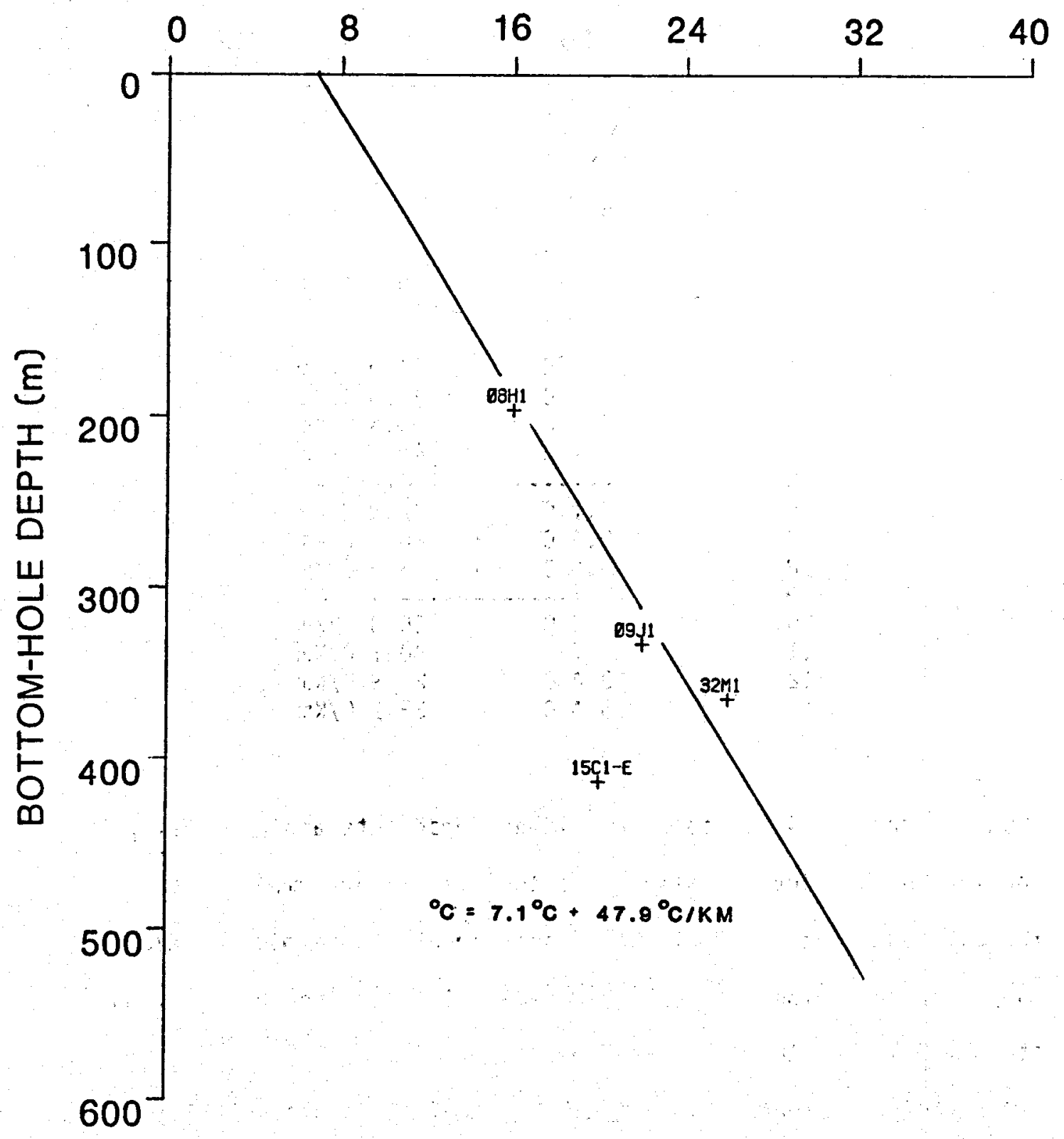

FIGURE 3 PLOT OF BOTTOM-HOLE TEMPERATURE VS.

BOTTOM-HOLE DEPTH FOR WELL DATA GROUP 1 


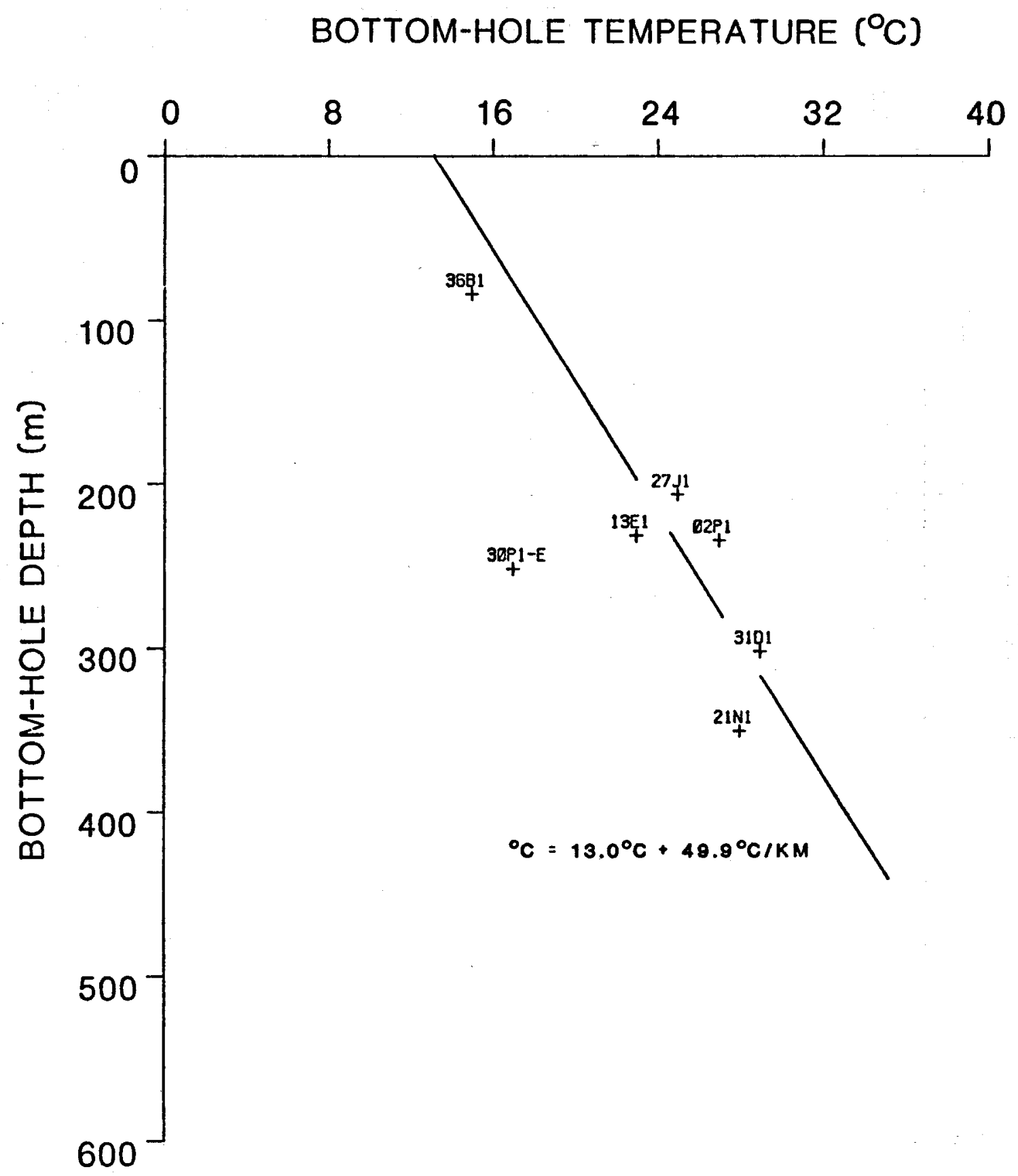

FIGURE 4 PLOT OF BOTTOM-HOLE TEMPERATURE VS. BOTTOM-HOLE DEPTH FOR WELL DATA GROUP 2 


\section{BOTTOM-HOLE TEMPERATURE $\left({ }^{\circ} \mathrm{C}\right)$}

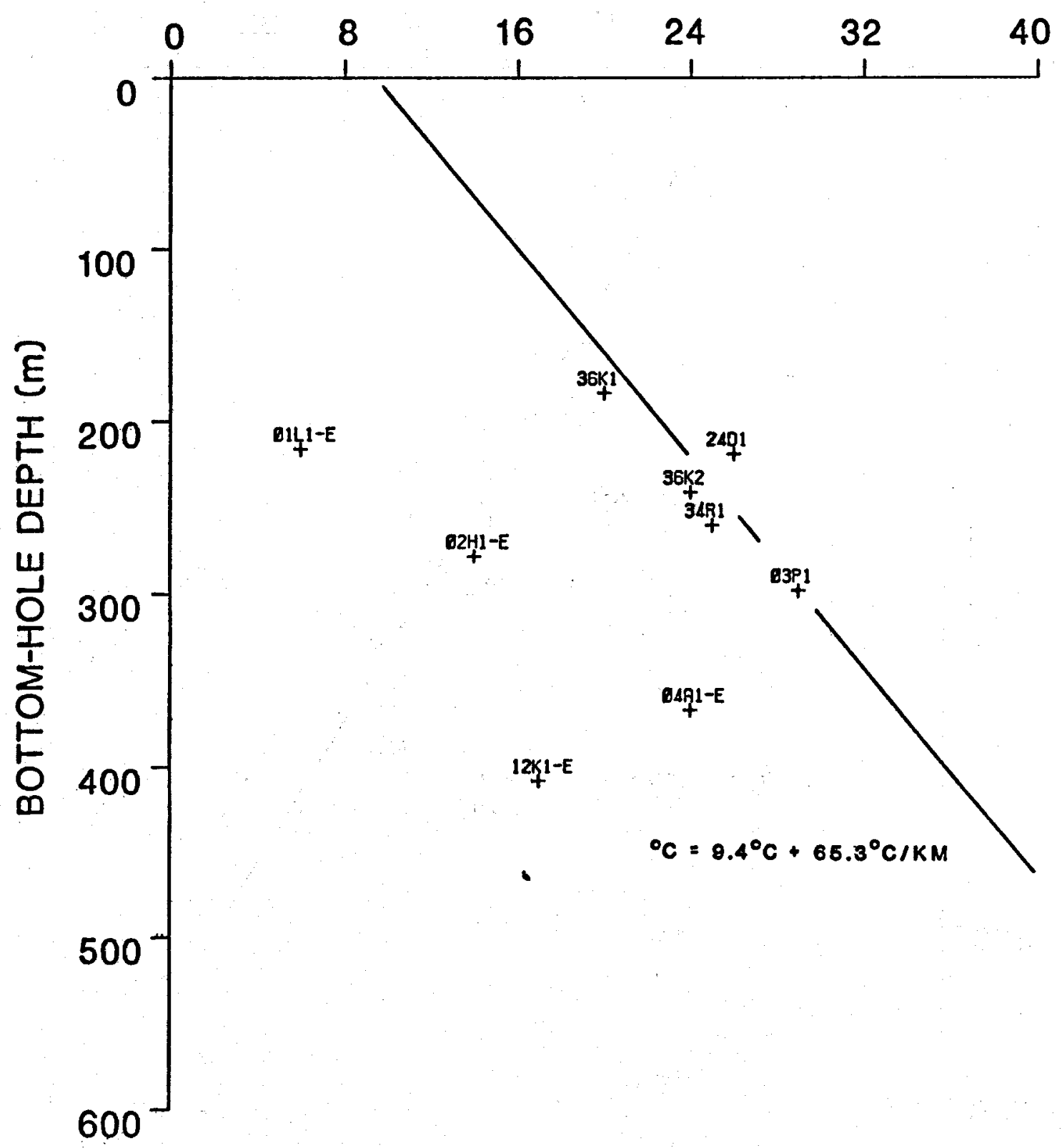

FIGURE 5 PLOT OF BOTTOM-HOLE TEMPERATURE VS. BOTTOM-HOLE DEPTH FOR WELL DATA GROUP 3 


\section{BOTTOM-HOLE TEMPERATURE $\left({ }^{\circ} \mathrm{C}\right)$}

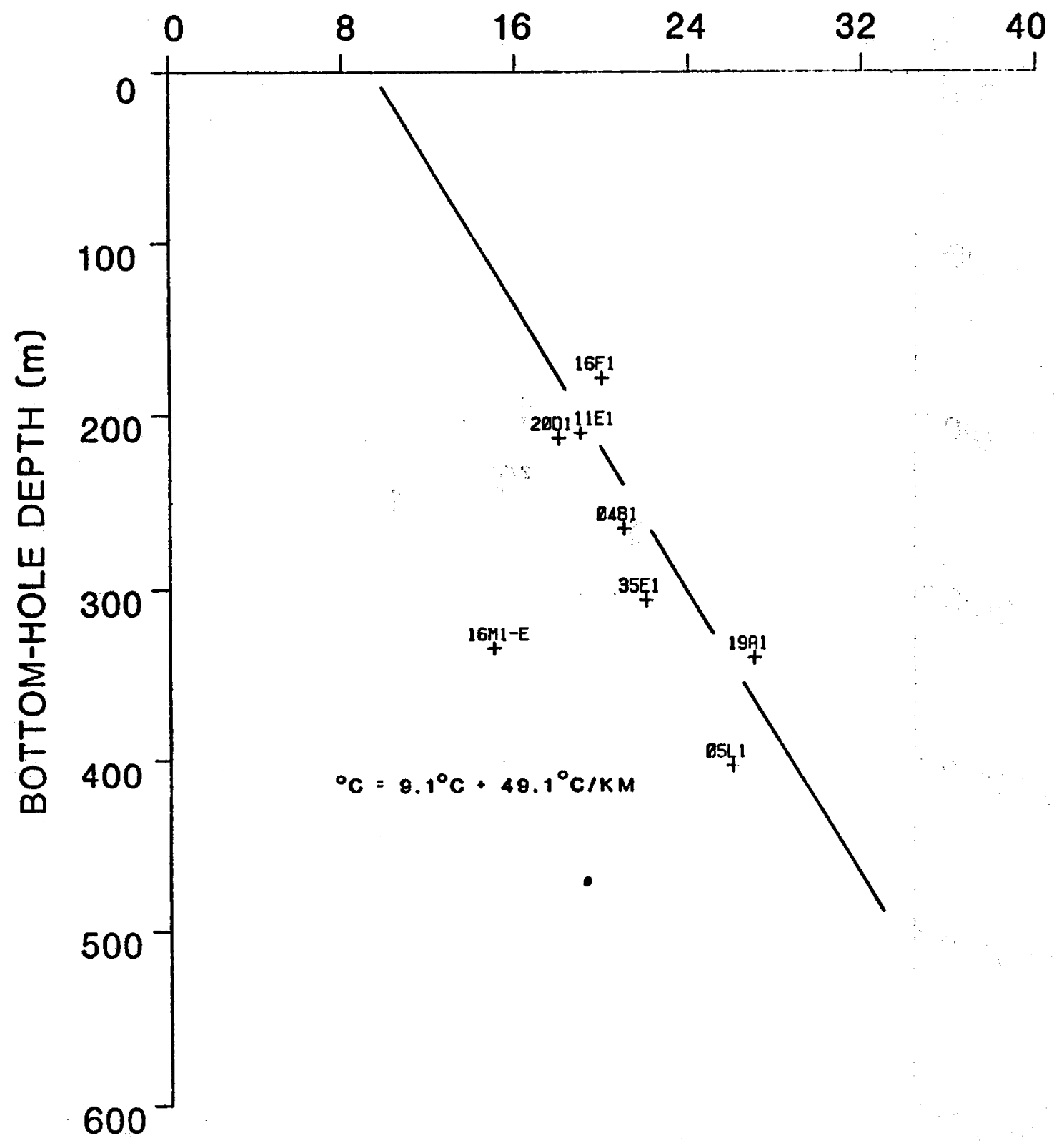

FIGURE 6 PLOT OF BOTTOM-HOLE TEMPERATLRE VS.

BOTTOM-HOLE DEPTH FOR WELL DATA GROUP 4 


\section{BOTTOM-HOLE TEMPERATURE $\left(^{\circ} \mathrm{C}\right)$}

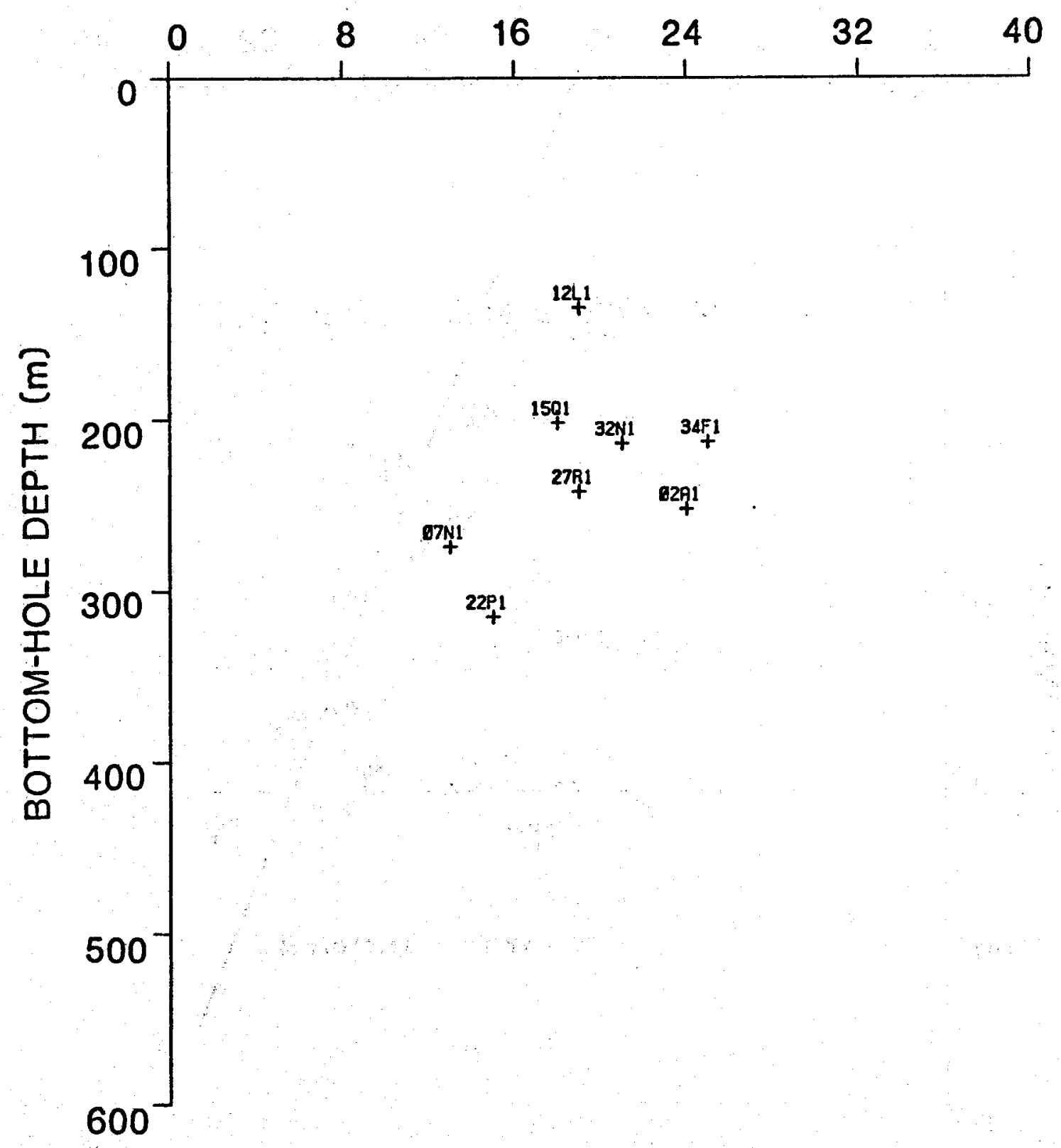

FIGURE 7 PLOT OF BOTTOM-HOLE TEMPERATURE VS. BOTTOM-HOLE DEPTH FOR WELL DATA GROUP 5 


\section{BOTTOM-HOLE TEMPERATURE ( $\left.{ }^{\circ} \mathrm{C}\right)$}

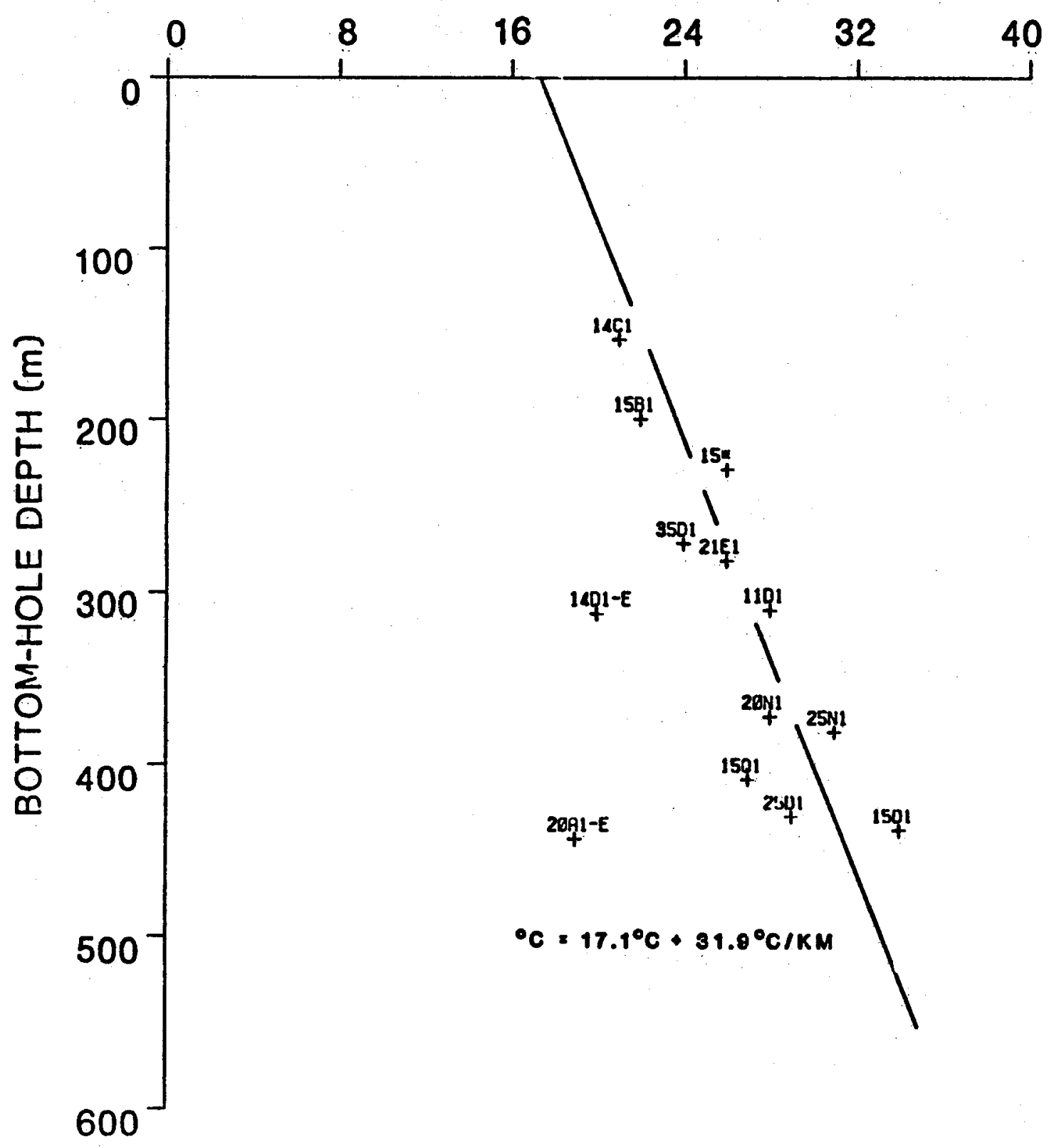

FIGURE 8 PLOT OF BOTTOM-HOLE TEMPERATURE VS.

BOTTOM-HOLE DEPTH FOR WELL DATA GROUP 6 
BOTTOM-HOLE TEMPERATURE ( $\left.{ }^{\circ} \mathrm{C}\right)$

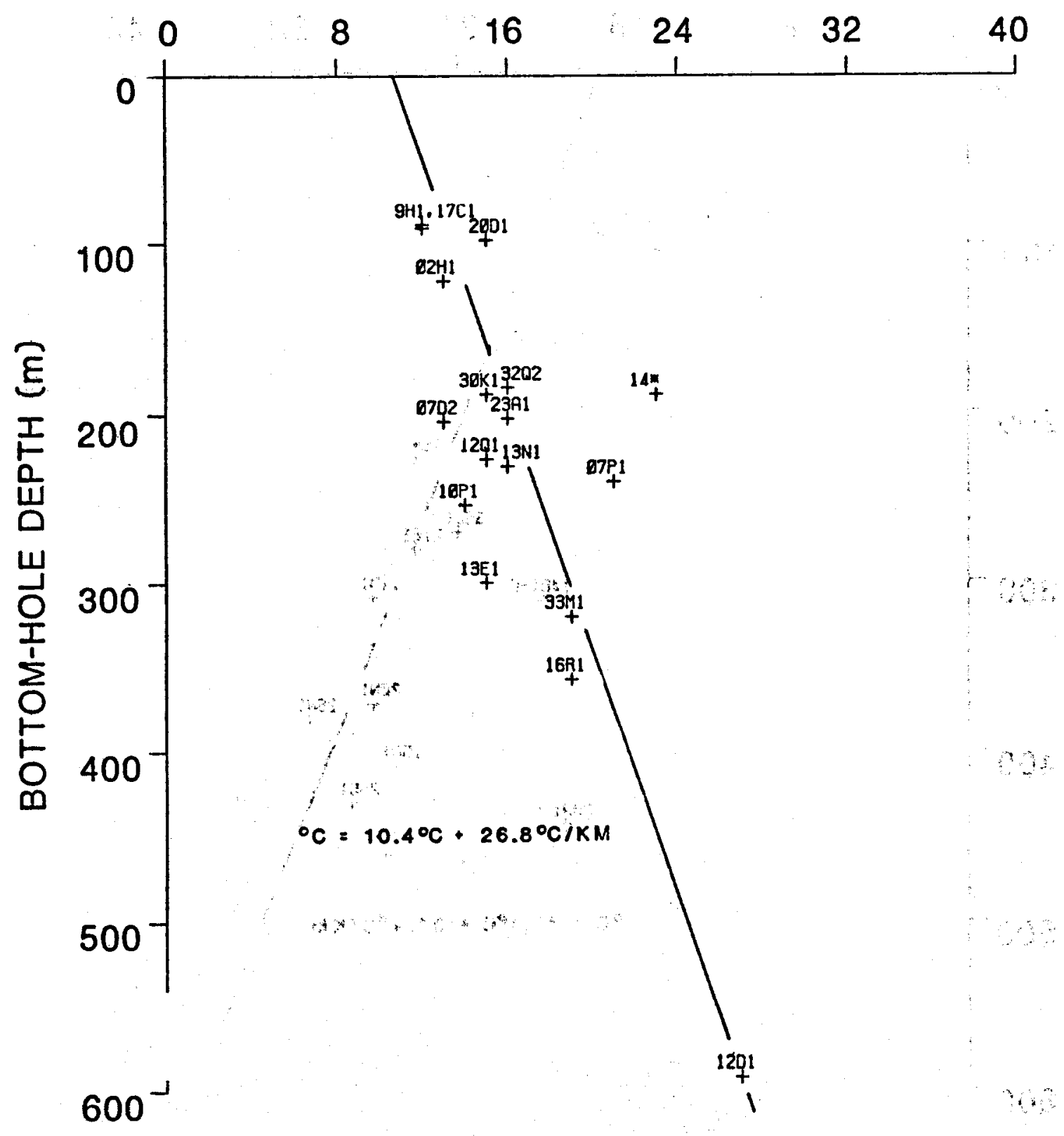

FIGURE 9 PLOT OF BOTTOM-HOLE TEMPERATURE VS.

BOTTOM-HOLE DEPTH FOR WELL DATA GROUP 7 
BOTTOM-HOLE TEMPERATURE $\left({ }^{\circ} \mathrm{C}\right)$

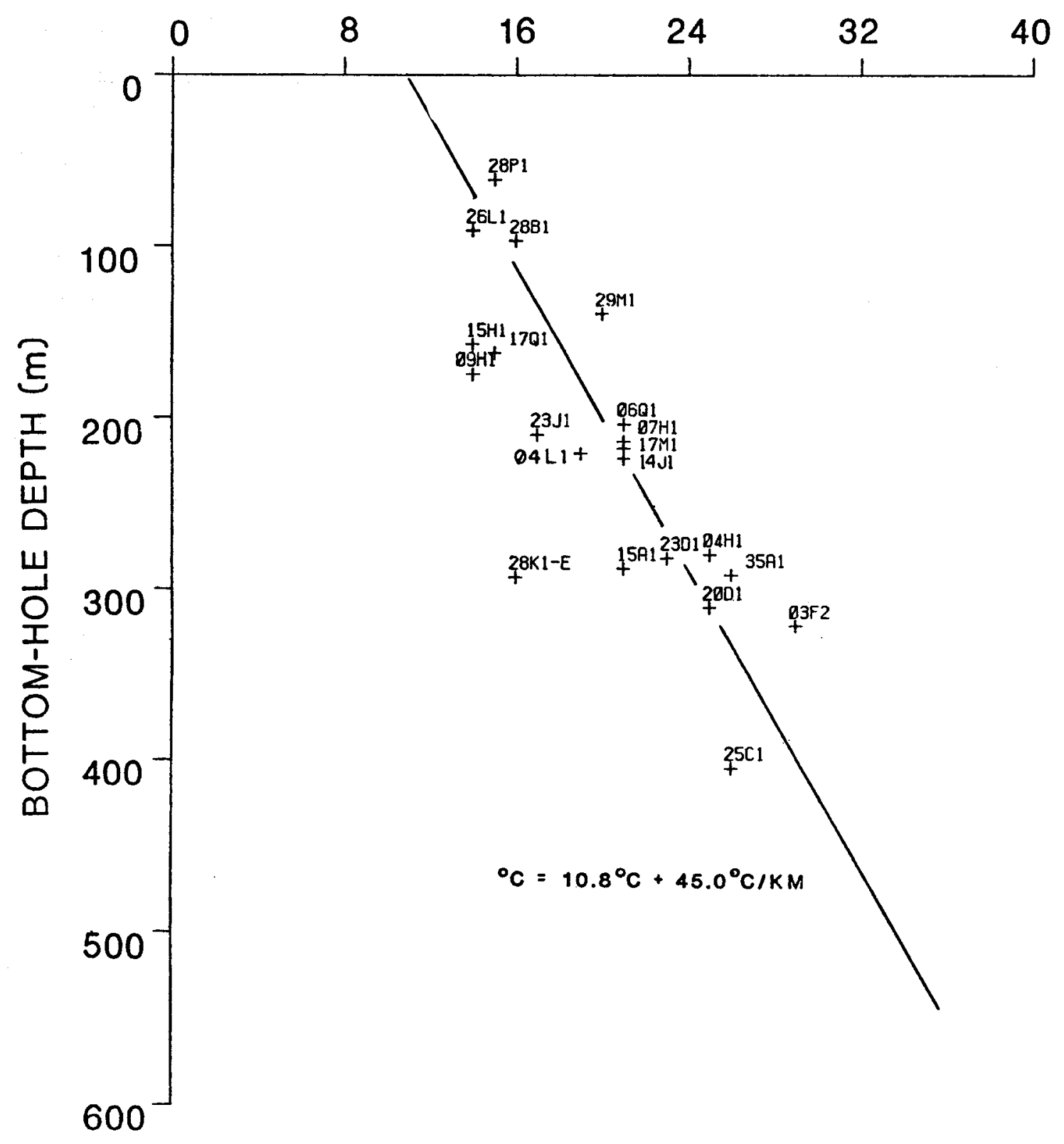

FIGURE 10 PLOT OF BOTTOM-HOLE TEMPERATURE VS.

BOTTOM-HOLE DEPTH FOR WELL DATA GROUP 8 
BOTTOM-HOLE TEMPERATURE $\left({ }^{\circ} \mathrm{C}\right)$

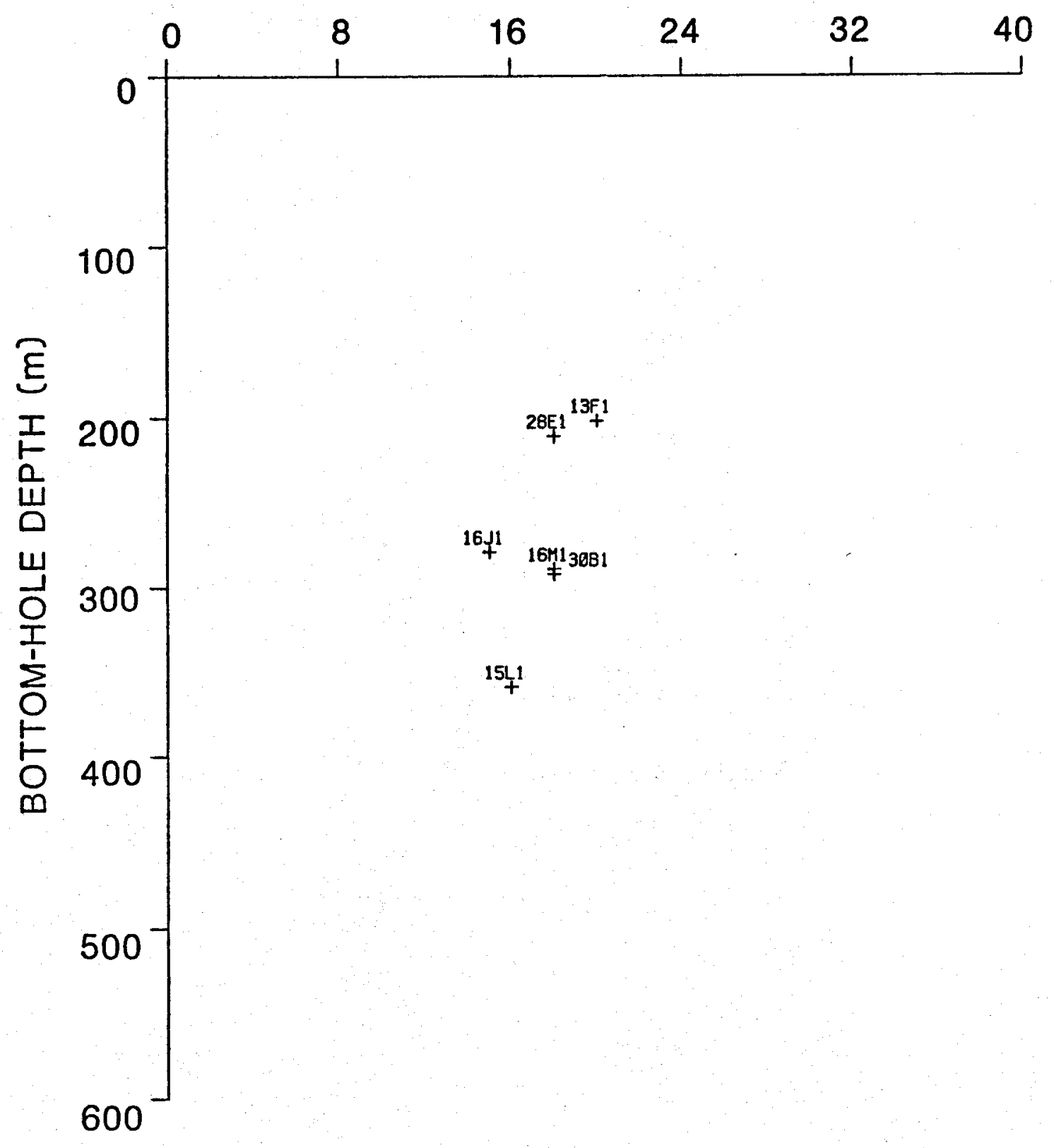

FIGURE 11 PLOT OF BOTTOM-HOLE TEMPERATURE VS. BOTTOM-HOLE DEPTH FOR WELL DATA GROUP 9 
BOTTOM-HOLE TEMPERATURE $\left({ }^{\circ} \mathrm{C}\right)$

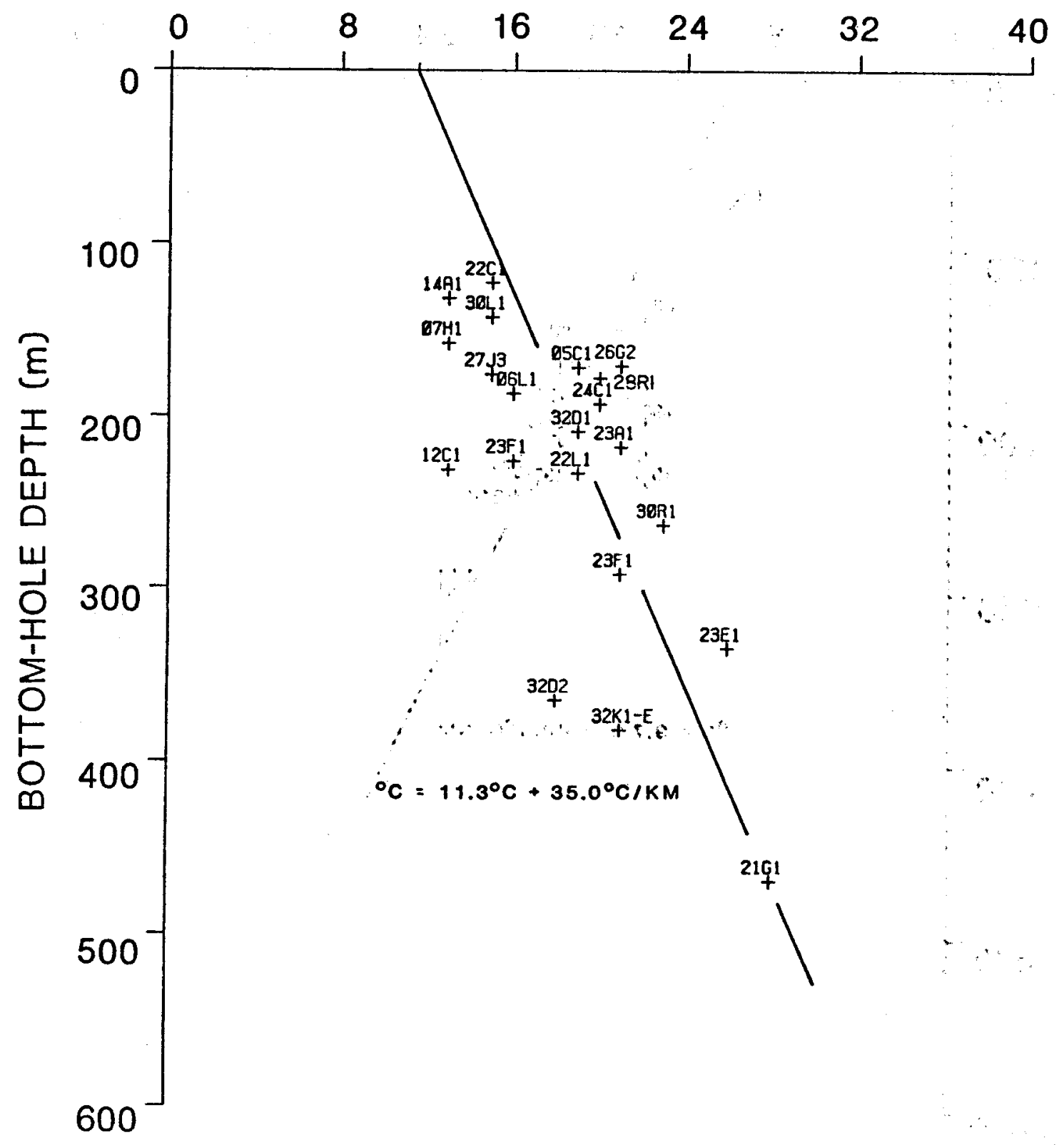

FIGURE: 12 PLOT OF BOTTOM-HOLE TEMPERATURE VS. 


\section{BOTTOM-HOLE TEMPERATURE ( $\left.{ }^{\circ} \mathrm{C}\right)$}

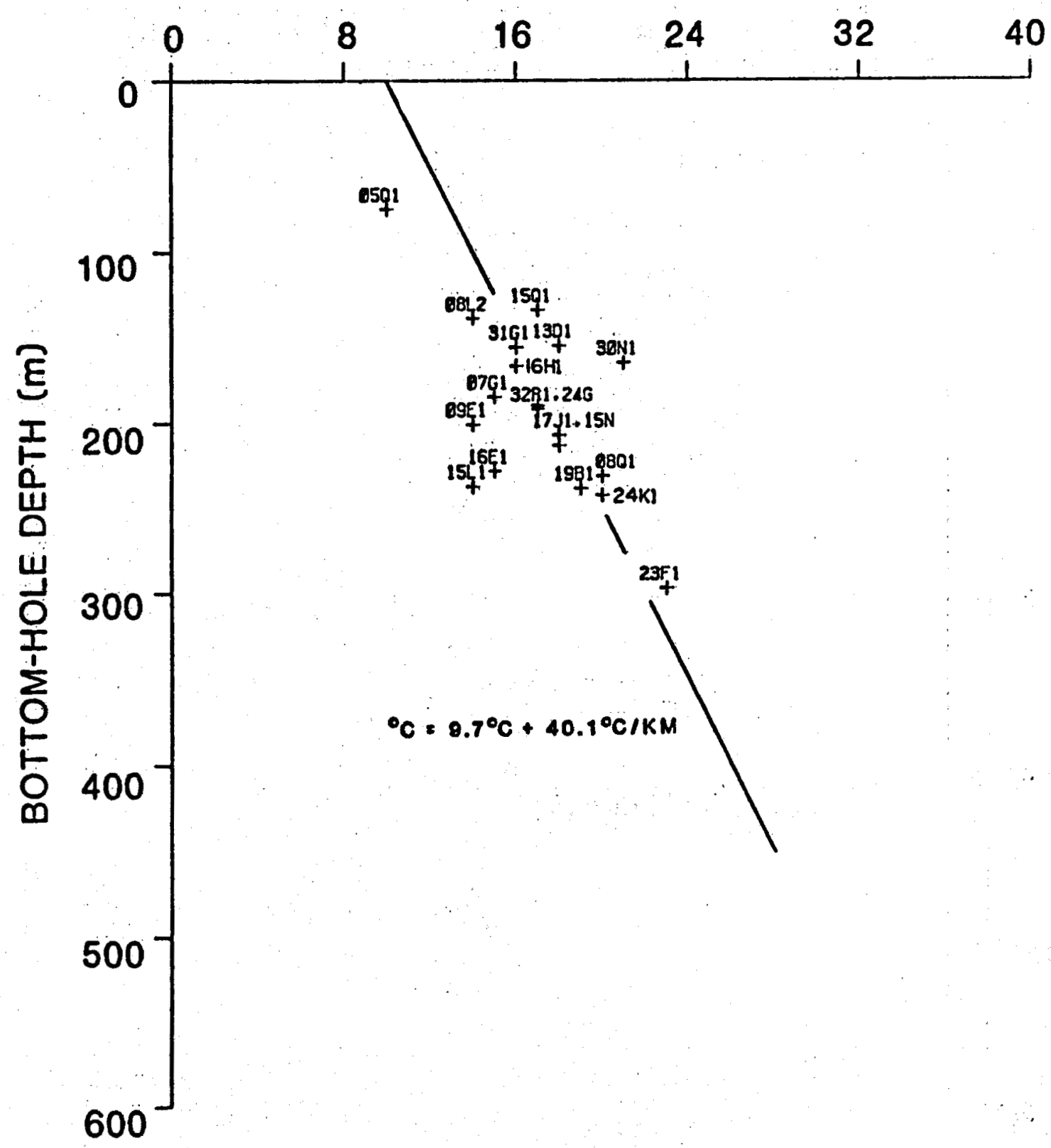

FIGURE 13 PLOT OF BOTTOM-HOLE TEMPERATURE VS. 
BOTTOM-HOLE TEMPERATURE $\left({ }^{\circ} \mathrm{C}\right)$

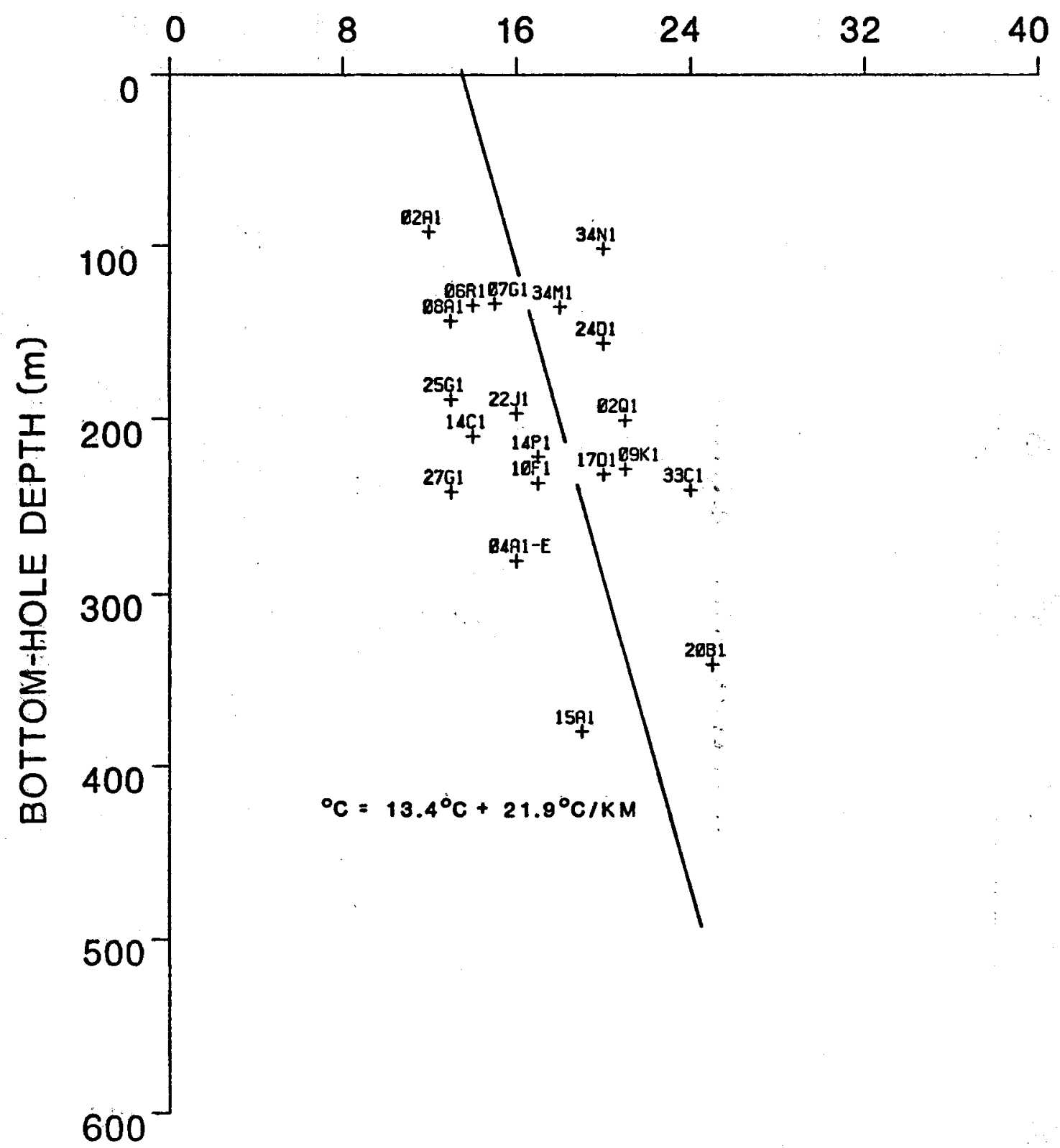

FIGURE 14 PLOT OF BOTTOM-HOLE TEMPERATURE VS. BOTTOM-HOLE DEPTH FOR WELL DATA GROUP 12 


\section{BOTTOM-HOLE TEMPERATURE $\left({ }^{\circ} \mathrm{C}\right)$}

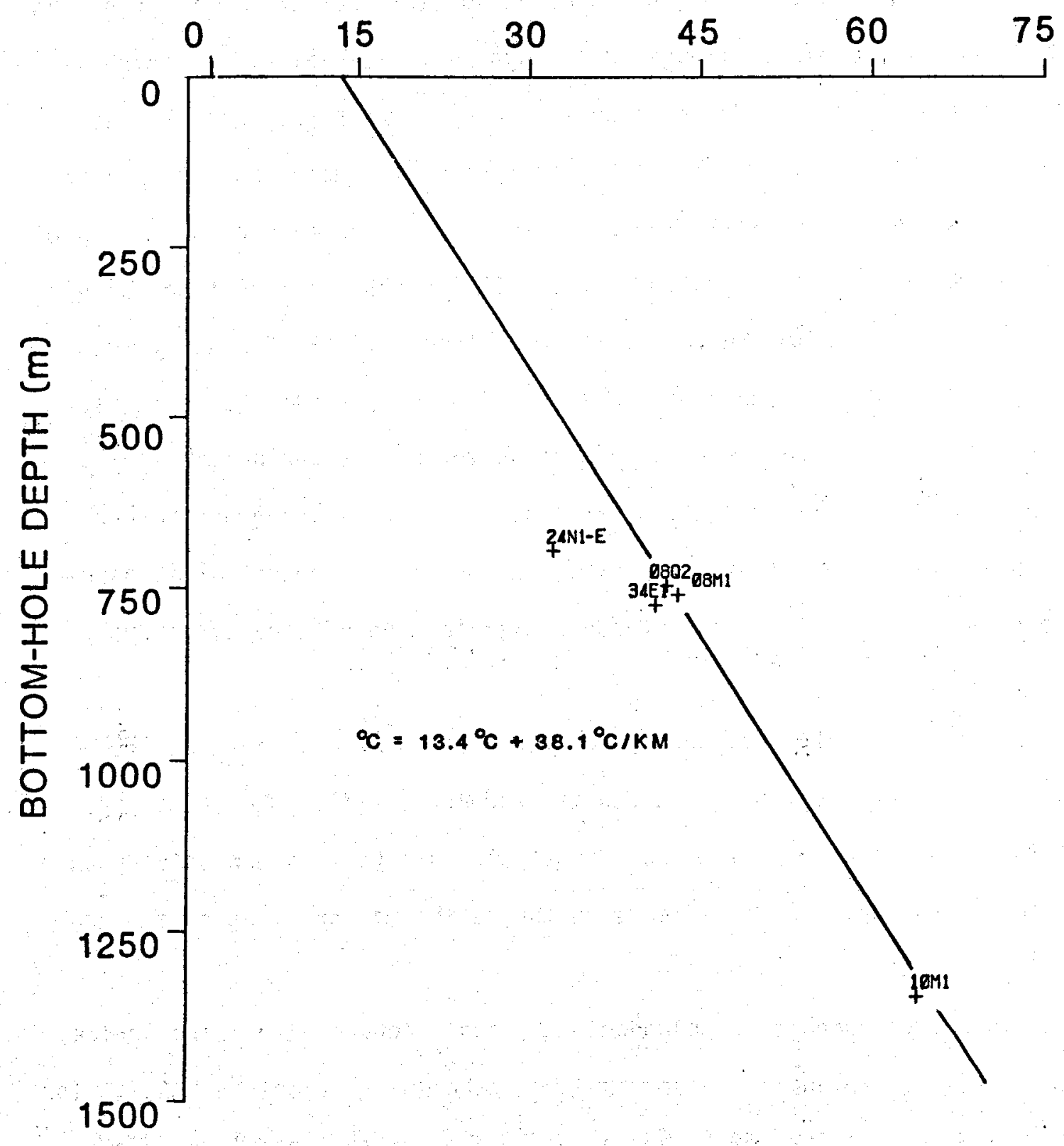

FIGURE 15 PLOT OF BOTTOM-HOLE TEMPERATURE VS. BOTTOM-HOLE DEPTH FOR DEEP WELL DATA GROUP 13 
It is likely there exists a slight difference in tool response between reporting agencies, which would account for the scattered data points in the BHT vs. BHD plots (figures 3 through 15). Several other controls may introduce error into the results. A well which has recently been pumped yields a higher BHT, and associated geothermal gradient because of a substantial vertical flow component and the subsequent upwelling of warmer water. Heat generated by the drilling process itself may significantly increase the BHT. Static water levels within the study area vary as much as $25 \mathrm{~m}$ annually (Luzier and others. 1968). This annual fluctuation is a manifestation of intensive pumpage during the irrigation season. Cyclic pumpage creates a non-steady state ground water flow system. The non-steady redistribution of nead potential is assumed to be associated with a nun-steady redistribution of convective heat potential.

In 1968, nearly 7,000 acre feet were pumped from T 16N.. R. 32E. which includes most of WDG 6 (Luzier and Burt, 1974). WDG 6. in the future, may shed some light on the relationship between excessive ground water.withdrawal and its effects on the geothermal gradient in a given flow system.

Intensive pumpage, compounded with local recharge from Lind Coulee. are possible explanations for the low geothermal gradient in WDG 7 . In figure 9 (WDG 7). the distribution of data about the calculated geothermal gradient indicates the actual gradient may vary from that which is reported.

The Moses Lake area (WDG 8) shows a favorable gradient for utilizing warmer water from depth. Scatter in the BHT VS. BHD plot (figure 10) may be a result of cyclic pumping. 
A reasonable geothermal gradient could not be fit to the data within WDG 9. The location coincides with anomalies in the piezometric surface. 8,000 acre feet were pumped from T. 19 N.. R. 30E. during the year of 1968 (Luzier and Burt, 1974). Intensive pumpage and recharge from the East Low Canal have been cited as possible explanations for this piezometric anomaly (Lobdell and Brown, 1977). The apparent low geothermal gradient in figure 11 (WDG 9) could be the result of this activity. WDG's in the northern portion of the study area (WDG 10 through WDG 12) display favorable geothermal gradients. It should be noted that there are substantial static water level declines within these areas: WDG 13: (figure 15) consists of those wells within the study area which are greater than $600 \mathrm{~m}$ in depth. The gradient, at $38.1^{\circ} \mathrm{C} / \mathrm{Km}$, is relatively low, below the average for the region. Biggane (1982) had noted that deeper wells in his study area showed a lower than average geothermal gradient, and suggested that this lower gradient may be ${ }^{\circ}$ attributed to a change in thermal conductivity at depth. For this study, however. 4 of the 5 wells which comprise WDG 13 fall within low gradient well data groups defined by shallower wells (1.e., groups 6, 10, and 11)." The other well occurs in the relatively high gradient area WDG 1. which also has a very low projected surface temperature. The gradient as determined by that average projected temperature and the BHT is $47.5^{\circ} \mathrm{C} / \mathrm{Km}$, close to the average for WDG 1.

The deep wells within this study area therefore, seem to reflect the average gradients for the well data groups. They may not be representative of the regional gradient because they are located in low gradient well data groups. 
Any one of the WDG's is a candidate for low-temperature geothermal development, provided a well is properly constructed. It would be unwise to select a low-temperature geothermal site without obtaining temperature logs from all of the wells in the nearby vicinity and analyzing them in great detail. 
Biggane, J. H., 1982. The low-temperature geothermal resource and stratigraphy of portions of Yakima County. Washington: Washington State Department of Natural Resources, Division of Geology and Earth Resources, Open-File Report 82-6, 01ympia. Washington.

Brown, J. C.. 1978, Discussion of geology and ground-water hydrology of the Columbia Plateau. with specific analysis of the Horse Heaven, Sagebrush Flat, and Odessa-Lind areas. Washington: Washington State Univ.. College of Engineering. Research Report 78/15-23, Pullman, Washington.

Lobde11, G. T., and Brown. J. C.. 1977. Geophysical investigations of Washington's ground-water resources, annual report 1976/1977: Washington State Univ.. College of Engineering. Research Report 77/15-76. Pullman, Washington.

Luzier. J. E.. and Burt, R. J.. 1974. Hydrology of basalt aquifers and depletion of ground-water in east-central Washington: Washington State Dept. of Ecology. Water-Supply Bulletin 33, 0lympa, Washington.

Tanaka, H.. Barrett, G.. and Wildrick, L., 1979, Regional basalt hydrology of the Columbia Plateau in Washington: Rockwell Hanford Operations, Report RHO-BWI-C-60, Richland, Washington. 